\section{Communicating new policy on antibiotic prophylaxis with patients: a randomised controlled trial}

\author{
S. Soheilipour, ${ }^{* 1,2}$ S. M. Dunne, ${ }^{3}$ C. Dickinson, ${ }_{1}^{4}$ S. E. Jabbarifar ${ }^{5}$ \\ and J. T. Newton ${ }^{6}$
}

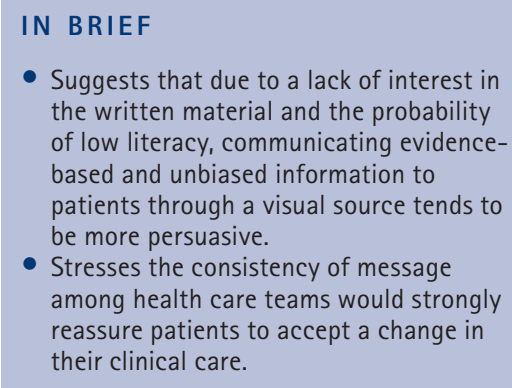

Objectives This trial aimed to assess the effectiveness of two different communication tools on the levels of anxiety and concern when a change in patients' treatment was introduced. Method Patients previously advised to have antibiotic prophylaxis before their dental treatments were randomised to receive information about the new policy either through a video accompanied by a written leaflet or just the leaflet. All patients completed a questionnaire to assess anxiety and concern as well as intentions regarding accepting dental treatment without antibiotic prophylaxis at enrolment point, after intervention and after meeting the cardiologist. Results Ninety questionnaires were analysed (45 in each group). The mean level of anxiety and concern scores were significantly reduced after the intervention point $(p<0.05)$. The ANOVA model revealed a significant reduction in the levels of anxiety and concern during the trial $(p<0.001)$. However, the main effect of group (intervention versus control) and the interaction term were not significant. At the end of trial there was no difference in the number of patients accepting dental treatment without cover in the two groups. Conclusion Patients appear more likely to accept a change if it is communicated directly to them by their practitioners via face to face consultation compared with video or leaflet. When there is a lack of time for in-depth consultation, video could be a more effective method than leaflet alone.

\section{INTRODUCTION}

Within a patient-centred philosophy of care patients should have the opportunity to make informed decisions about their care and treatment, in partnership with their healthcare professionals. ${ }^{1}$ They need high quality information with which they can actively participate in their care, make an informed decision, come to a common understanding with their physicians and comply more fully with treatment requirements. ${ }^{2}$ This is

\footnotetext{
Research collaborator, King's College London, Dental Public Health Research group, London, UK; ${ }^{2}$ Assistant Professor, Department of Oral Public Health and TorabiNejad Dental Research Center, Dental School, Isfahan University of Medical Sciences, Isfahan, Iran; ${ }^{3}$ Professor in Primary Dental Care, Department of Primary Dental Care, King's College London, London, UK; ${ }^{4}$ Consultant in Sedation and Special Care Dentistry, Guy's and St Thomas NHS Foundation Trust, Guy's Hospital, London, UK; ${ }^{5}$ Professor in Pediatric Dentistry, Department of Pedodontics and Torabi Nejad Dental Research Center, School of Dentistry, Isfahan University of Medical Sciences, Isfahan, Iran; ${ }^{6}$ Professor of Psychology as Applied to Dentistry, Division of Health and Social Care Research, King's College London, London, UK

${ }^{*}$ Correspondence to: Shimae Soheilipour

Email: shimae.soheilipour@kcl.ac.uk,

Tel: +442073463481
}

\section{Online article number E5}

Refereed Paper - accepted 13 May 2013

DOI: 10.1038/sj.bdj.2013.742

${ }^{\oplus}$ British Dental Journal 2013; 215: E5 particularly important when a substantive change from current practice needs to be applied to their treatment.

Communicating evidence-based and unbiased information through a credible source tends to be more persuasive. Source credibility is high when the source is perceived as knowledgeable and trusted. Patient education should be an integral part of communication between health providers and patients. In the traditional way, the clinician can verbally provide patients with relevant information as a component of informed consent. Although the doctorpatient interaction must remain at the centre of the process, varying communication abilities and insufficient time discussing treatment may result in patients having an inadequate understanding of the evidence. Health professionals can provide patient education through a variety of other means like educational materials such as leaflets and, more recently, websites..$^{2-4}$

Evidence-based consumer information designed for patients can increase patient understanding of the evidence for a change. It can also facilitate the implementation of change in clinical practice by reducing possible concerns and eliciting patients' preferences. ${ }^{2}$ Nowadays, decision-aids for a variety of clinical processes are being developed, however, there is uncertainty regarding their efficacy in communicating information and effectiveness in helping patients make decisions. ${ }^{5}$ Mixed results have been shown by studies investigating the effectiveness of evidence-based leaflets as a communication tool to improve information to patients. ${ }^{6,7}$ Many patients do not read such forms and of those who do, many do not fully comprehend the information provided. Written information requires at least basic literacy as well as the motivation to read the material provided. To overcome the deficiencies of information leaflets, electronic media such as audiotape, videotape, and interactive DVDs have been recommended. ${ }^{3}$

The new guideline published in the UK by the National Institute for Health and Clinical Effectiveness (NICE) in 2008 abolished the requirement to give antibiotic prophylaxis (AP) to patients at risk of infective endocarditis. ${ }^{1}$ The results of current studies on compliance with the guideline among health professionals in the UK showed that most of them are aware of the guideline, however, their compliance is varied..$^{8,9}$ It seems hard 
to explain the substantive shift in policy to patients who have been reminded for years how important their antibiotic cover was. Studies investigating attitudes on implementation of the new NICE guideline revealed various levels of concern among patients. ${ }^{10,11}$ Clear information concerning the reason for the changes in current practice is necessary to be communicated effectively for patient reassurance.

This trial aimed to investigate the impact of an information video, provided in addition to written and verbal information, on the anxiety level and decision making process to accept dental treatment without antibiotic prophylaxis among patients at risk of infective endocarditis who have received antibiotic prophylaxis for dental procedures in the past. The null hypothesis was that supplementing written information with a video presentation informing patients of the rationale for the change in antibiotic prophylaxis prescribing policy given by a specialist dentist would have no impact on patients' degree of anxiety about confidence in and willingness to undergo dental treatment without antibiotic prophylaxis in comparison to a leaflet alone.

\section{METHODS}

\section{Participants}

A consecutive series of patients attending the outpatient clinics in the Adult Congenital Heart Disease Service at the St Thomas Hospital, London, were approached. In order to be eligible to participate patients were required to have a heart problem that would have necessitated antibiotic prophylaxis to prevent infective endocarditis as a result of dental treatment according to the previous guidelines. They must be able to comprehend the written materials (patient information leaflets, consent forms and questionnaires). Patients were excluded if they had already undergone dental treatment without prophylaxis following the change in guidance.

A sample size of 45 per group was identified as sufficient to detect a medium effect size of 0.6 for the anxiety and concern scales in two groups that was compared using the t-test with equal variance and a significance level of 0.05 and $80 \%$ power. This effect size

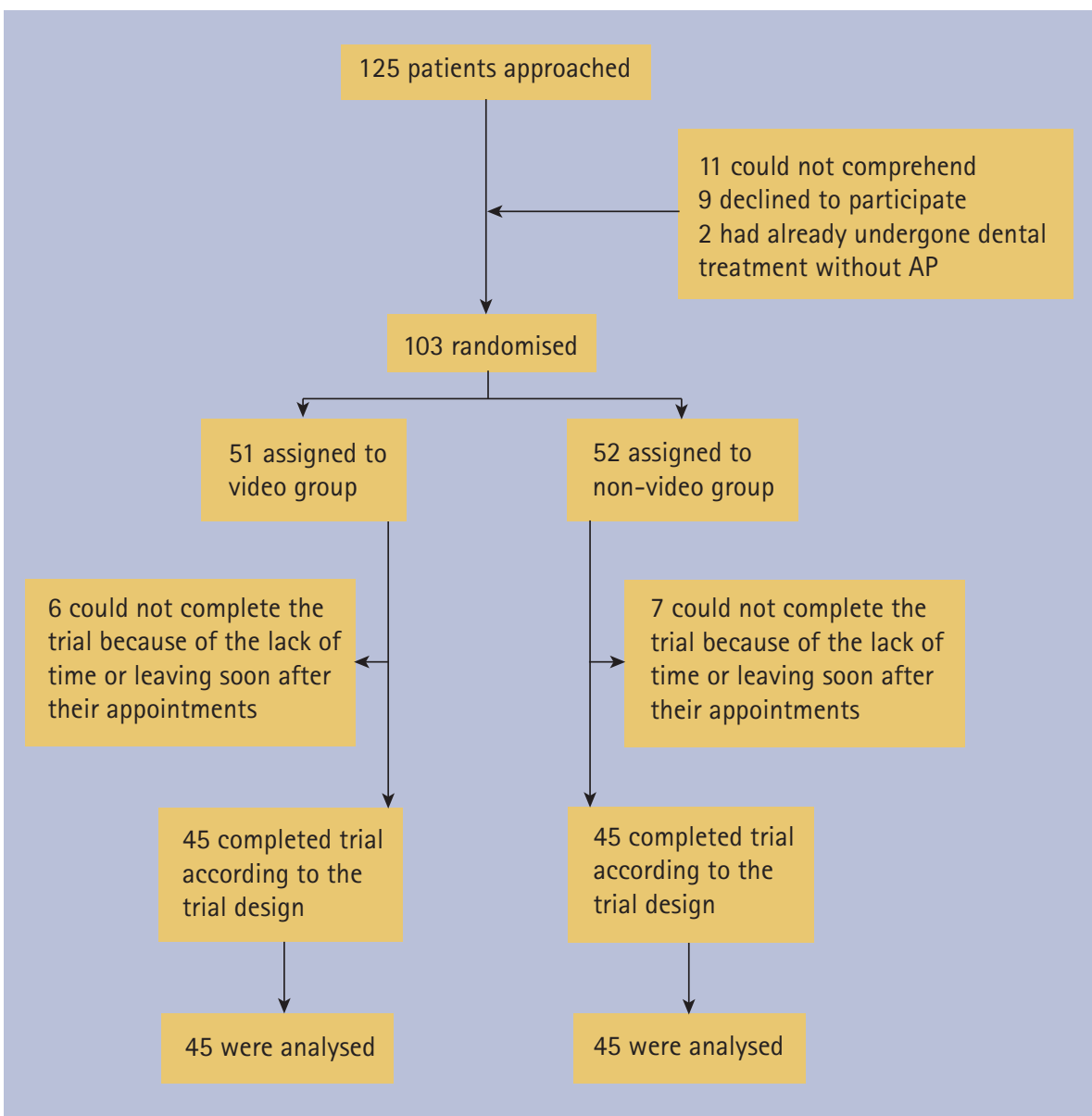

Fig. 1 Trial profile showing total number of individuals approached and participant numbers throughout the study

was judged to be clinically significant by the research team and would equal a $60 \%$ difference in mean ratings of anxiety and concern on a five-point scale, in the absence of previously published information on the anticipated differences between the two groups (calculations performed using GPower 3.1 for Mac).

\section{Measures}

A two part questionnaire was designed for data collection. The first part asked about demographic information; heart conditions and self-rated general and dental health of patients. The second part of the questionnaire intended to investigate patients' feelings and intentions when they were informed by their dentist that they no longer needed to take antibiotics before dental treatment. The first two questions assessed their level of anxiety and concern on a visual analogue score between 0 and 10. The next three closed questions asked them about their intentions to take antibiotic prophylaxis based on the knowledge that they have about the new policy at each stage in the trial.
Patients were asked to complete the questionnaire before any intervention (point A) to assess their anxiety and reactions at base line, immediately after the intervention (point B), and following their appointment with the consultant cardiologist (point C). The questionnaire was piloted on four patients before starting the main trial to assess feasibility, but no changes were made to the measures after the trial commenced. Data from the pilot study were not included in the main analysis.

\section{Intervention}

The randomised controlled trial (RCT) reported here was conducted as part of a multi-stage project investigating the barriers and facilitating factors on the implementation of the new guideline concerning antibiotic prophylaxis. Earlier qualitative studies $^{10,12}$ by the researchers revealed that cardiologists and dentists were the professional groups who patients would mostly believe. In this study the information about the change in antibiotic prophylaxis based on the NICE guideline and the relevant evidence were communicated with 


\begin{tabular}{|c|c|c|c|c|}
\hline & & $\begin{array}{l}\text { Non-video group } \\
(n=45)\end{array}$ & $\begin{array}{l}\text { Video group } \\
(n=45)\end{array}$ & Comparison \\
\hline Age & $\begin{array}{l}\text { Mean (SD) } \\
\text { Range }\end{array}$ & $\begin{array}{l}32.60(3.6) \\
16-68\end{array}$ & $\begin{array}{l}28.51(9.57) \\
16-59\end{array}$ & $t=1.65, p=0.10$ \\
\hline Gender & $\mathrm{M} / \mathrm{F}$ & $25 / 20$ & $23 / 22$ & $\mathrm{Chi}^{2}=0.18, p=0.83$ \\
\hline Ethnicity* & British/Others & $39 / 5$ & $37 / 7$ & $\mathrm{Chi}^{2}=0.34, \mathrm{p}=0.77$ \\
\hline Education* & $\begin{array}{l}\text { Up to } 0 \text { level (CSE, GCSE) } \\
\text { A levels } \\
\text { University degree } \\
\text { Postgraduate degree } \\
\text { Others }\end{array}$ & $\begin{array}{l}19 \\
10 \\
6 \\
4 \\
1\end{array}$ & $\begin{array}{l}16 \\
9 \\
12 \\
3 \\
0\end{array}$ & $\mathrm{Chi}^{2}=0.75, p=0.69$ \\
\hline Medical status** & $\begin{array}{l}\text { Cardiac pacemakers and implanted defibrillator } \\
\text { Previous coronary artery bypass graft surgery } \\
\text { Prosthetic heart valves } \\
\text { Surgically constructed systemic pulmonary shunts or conduits } \\
\text { Surgical correction of septal defect (Hole on the heart) } \\
\text { Mitral valve prolapse } \\
\text { Previous bacterial endocarditis } \\
\text { Congenital heart disease }\end{array}$ & $\begin{array}{l}2 \\
1 \\
13 \\
3 \\
16 \\
4 \\
0 \\
25\end{array}$ & $\begin{array}{l}3 \\
2 \\
5 \\
5 \\
13 \\
1 \\
4 \\
30\end{array}$ & $\begin{aligned} \mathrm{Chi}^{2} & =0.21, \mathrm{p}=1.0 \\
\mathrm{Chi}^{2} & =0.35, \mathrm{p}=1.0 \\
\mathrm{Chi}^{2} & =4.44, \mathrm{p}=0.06 \\
\mathrm{Chi}^{2} & =0.55, \mathrm{p}=0.71 \\
\mathrm{Chi}^{2} & =0.46, \mathrm{p}=0.65 \\
\mathrm{Chi}^{2} & =1.91, \mathrm{p}=0.36 \\
\mathrm{Chi}^{2} & =4.19, \mathrm{p}=0.12 \\
\mathrm{Chi}^{2} & =1.17, \mathrm{p}=0.39\end{aligned}$ \\
\hline General health & $\begin{array}{l}\text { Poor } \\
\text { Fair } \\
\text { Good } \\
\text { Excellent }\end{array}$ & $\begin{array}{l}1 \\
10 \\
28 \\
6 \\
\end{array}$ & $\begin{array}{l}3 \\
3 \\
32 \\
7 \\
\end{array}$ & $\mathrm{Chi}^{2}=5.11, p=0.16$ \\
\hline Oral health & $\begin{array}{l}\text { Poor } \\
\text { Fair } \\
\text { Good } \\
\text { Excellent }\end{array}$ & $\begin{array}{l}2 \\
9 \\
26 \\
8\end{array}$ & $\begin{array}{l}3 \\
5 \\
32 \\
5\end{array}$ & $\mathrm{Chi}^{2}=2.66, p=0.45$ \\
\hline
\end{tabular}

patients through two different methods: leaflet (written information) and leaflet plus videotape (watching a dialogue given by a consultant in special care dentistry about the new guideline). Both groups received confirmation about the change from a cardiologist during the last stage of the study.

\section{Design of study}

Before randomisation, all patients were given written information explaining the purpose and requirements of the study. Informed consent for the trial was obtained in writing. Participants were enrolled and allocated to arms of the trial by a single researcher (SS). Patients were assigned to the video or non-video group following a simple random allocation sequence drawn up by an independent statistician using random number tables. The allocation sequence was concealed using sealed opaque envelopes, prepared in advance. The allocation ratio of intervention to control was $1: 1$. There were no changes to the protocol following commencement of the trial.

Patients in the non-video group received a three-page leaflet produced by Guy's and St Thomas NHS Foundation Trust to inform patients about changes in the use of antibiotics for the prevention of endocarditis before dental treatment.

Patients in the video group additionally watched a 5 min videotape. In the video, the information on the leaflet was presented verbally by a consultant in special care dentistry from Guy's and St Thomas NHS Foundation Trust with more emphasis on the evidence behind the new guideline and the importance of good oral health for the prevention of infective endocarditis.

Patients spent time in the waiting area reading the leaflet and then were approached by the researcher to complete the same questionnaire to assess their level of anxiety after intervention. In the next step, patients in both groups were asked to discuss the new policy with the cardiologist in the consultation meeting. The same questionnaire was completed by each patient after the visit.

Ethical approval for the trial was obtained from the King's College Hospital Research Ethics Committee (Reference number 08/H0808/126).

\section{MASKING}

Recruitment and intervention were provided by one person, so it was impossible to mask at these stages. Patients also became aware of the group they were in. However, at the stage of consultation meeting, cardiologists were blinded to which intervention had occurred. Data analysis was also masked; groups were coded to mask the groups during the analysis.

\section{ANALYSIS}

SPSS 17 was used for statistical analysis. Data for the two groups were analysed for comparability at baseline. The effect of the information video on the levels of anxiety and concern of the participants over time was analysed using repeated analysis of variance (ANOVA). Differences in intention were analysed with Chi-square tests and t-tests. A separate analysis was conducted for each outcome variable. Statistical significance was inferred at a two-tailed test level of $p<0.05$. No subgroup or adjusted analyses were performed.

\section{RESULTS}

Between June and September 2009, 125 patients were approached to join the trial. Twenty-two patients were excluded from the trial: eleven could 
Table 2 Anxiety and concern scores at different points of the trial, difference scores in the two groups and the results of ANOVA model

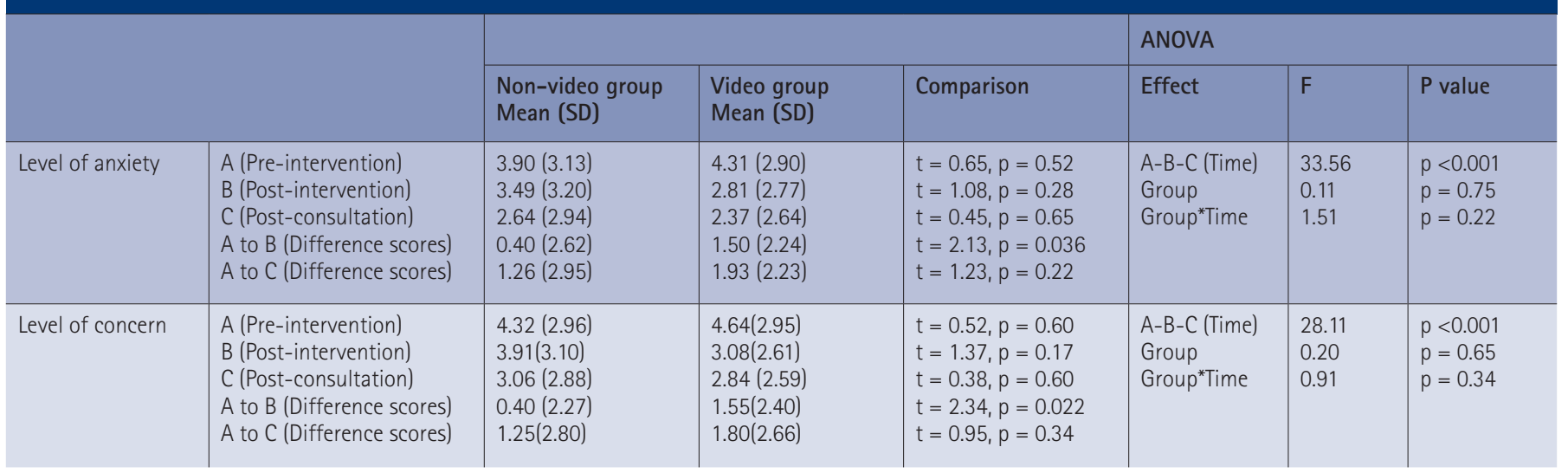

not comprehend the purpose of the trial; nine were not interested in participating and two had already undergone dental treatment without prophylaxis. The remaining 103 patients were enrolled. Fifty-one patients were assigned to the video group and 52 patients to the nonvideo group. Thirteen patients were also excluded during the trial (seven in the video group and six in the non-video group) due to the lack of time to complete all the stages. Final analysis was performed on the data from 90 patients (Fig. 1) according to the group originally assigned at randomisation. The trial ended when the target sample size of 90 complete participants was reached.

There were no differences in baseline characteristics between the groups (Table 1).

The effect of sex, ethnicity, level of education and type of heart condition on the anxiety and concern score at enrolment was examined by comparing groups using the Mann Whitney U test. Only one significant effect was found. Female patients reported significantly higher baseline levels of concern about becoming ill after dental treatment than male patients (mean 5.17 [SD = 3.11] vs. 3.88 [SD = 2.67]; $\mathrm{p}=0.037$ ).

To calculate the level of anxiety patients were asked 'How anxious do you feel about having the treatment without taking antibiotic cover?' There was no statistically significant difference between the two groups initially (Phase A). Anxiety level decreased over the study period in both the non-video and video group and more change was found in the video group after watching the video, however, no significant differences were found in the level of anxiety between the two groups in any stage of the trial $(\mathrm{A}, \mathrm{B}, \mathrm{C})(\mathrm{p}>0.05)$. The same results appeared when patients were asked to mark their level of concerns about becoming ill after the dental treatment (Table 2).

In order to explore changes in levels of anxiety and concern over time, difference scores were calculated for change in the levels of anxiety and concern across the possible change points (A to B; A to $\mathrm{C})$. Difference scores for anxiety and concern were significantly higher in the video group after watching the video (A to $\mathrm{B})$ compared with the non-video group (1.50 vs. $0.40, \mathrm{p}=0.036 ; 1.55$ vs. 0.40 . $\mathrm{p}=0.022$ respectively). However, the anxiety and concern scores at the end of trial did not change significantly for the non-video group and video group when compared with baseline levels (A to C).

The ANOVA model revealed a significant reduction in the levels of anxiety and concern during the trial $(\mathrm{p}<0.001)$. However, the main effect of group (intervention vs control) and the interaction term were not significant. The results are summarised in Table 2.

The other three questions in the questionnaire investigated patients' reactions when they were informed of the new guideline by a dentist. Table 3 presents the proportion of answers in two groups at different points in the trial.

The first question asked 'How likely is it that you would ask for an opinion from another doctor or dentist?' The number of patients who definitely would ask for another opinion decreased after watching the video, while the same reduction was reached in the non-video group after consultation with the cardiologist.

The next question asked about 'How likely is it that you would ask to go somewhere else for your treatment?' Patients in the non-video group were more likely to go somewhere else before having the opinion from their cardiologist. However, the difference wasn't significant.

In response to the final question; 'How likely is it that you would go ahead with the dental treatment without cover?' the number of patients who definitely would go ahead with treatment without cover, which was the same at baseline for both groups, was higher at point $B$ and $C$ in the video group.

No harmful or unintended effects were noted for either group.

\section{DISCUSSION}

Trials have attempted to evaluate the effectiveness of different designs of communication tools in a wide variety of clinical settings using a range of clinical problems and outcome measures. Consequently, it is difficult to generalise their results in all circumstances. In this study we assessed two different tools designed to inform patients about a change in policy on antibiotic prophylaxis before high risk dental procedures. The levels of anxiety and concern were not considered to be high at baseline among all participants in this study. The results demonstrated that both forms of communication (video/leaflet and leaflet alone) were effective in reducing patients' concerns and improved their intention to continue dental treatments without having antibiotic cover. However, the levels of anxiety and concern significantly reduced among patients in the video group compared with the non-video group before consultation. As recognised by previous studies, ${ }^{6,7}$ a lack of intention to read the leaflet or comprehension of the leaflet's 
Table 3 Number of respondents giving various answers to the questions investigated patients' reactions, when they were informed of the new guideline by a dentist

\begin{tabular}{|c|c|c|c|c|c|c|}
\hline \multirow[t]{2}{*}{ Answers } & \multicolumn{2}{|l|}{ A } & \multicolumn{2}{|l|}{ B } & \multicolumn{2}{|l|}{ C } \\
\hline & $\begin{array}{l}\text { Non-video group } \\
(\%)\end{array}$ & $\begin{array}{l}\text { Video group } \\
(\%)\end{array}$ & $\begin{array}{l}\text { Non-video group } \\
(\%)\end{array}$ & $\begin{array}{l}\text { Video group } \\
(\%)\end{array}$ & $\begin{array}{l}\text { Non-video group } \\
(\%)\end{array}$ & $\begin{array}{l}\text { Video group } \\
(\%)\end{array}$ \\
\hline \multicolumn{7}{|c|}{ 'How likely is it that you would ask for an opinion from another doctor or dentist?' } \\
\hline I definitely would ask & $10(22 \%)$ & $15(33 \%)$ & $10(22 \%)$ & $4(9 \%)$ & $5(11 \%)$ & $4(9 \%)$ \\
\hline I probably would ask & $12(27 \%)$ & $11(24 \%)$ & $7(16 \%)$ & $9(20 \%)$ & $9(20 \%)$ & $8(18 \%)$ \\
\hline I don't know whether I would ask or not & $10(22 \%)$ & $9(20 \%)$ & $7(16 \%)$ & $8(18 \%)$ & $6(13 \%)$ & $7(16 \%)$ \\
\hline I probably wouldn't ask & $12(27 \%)$ & $7(16 \%)$ & $15(33 \%)$ & $18(40 \%)$ & $18(40 \%)$ & $19(41 \%)$ \\
\hline I definitely wouldn't ask & $1(2 \%)$ & $3(7 \%)$ & $6(13 \%)$ & $6(13 \%)$ & $7(16 \%)$ & $7(16 \%)$ \\
\hline Comparison & \multicolumn{2}{|c|}{$\mathrm{Chi}^{2}=3.41, \mathrm{p}=0.49$} & \multicolumn{2}{|c|}{$\mathrm{Chi}^{2}=3.16, p=0.53$} & \multicolumn{2}{|c|}{ Chi2 $=0.17, p=0.99$} \\
\hline \multicolumn{7}{|c|}{ 'How likely is it that you would ask to go somewhere else for your treatment?' } \\
\hline I definitely would ask & $2(4 \%)$ & $6(13 \%)$ & $3(7 \%)$ & $2(4.5 \%)$ & $2(4.5 \%)$ & $3(7 \%)$ \\
\hline I probably would ask & $3(7 \%)$ & $4(9 \%)$ & $5(11 \%)$ & $2(4.5 \%)$ & $5(11 \%)$ & 0 \\
\hline I don't know whether I would ask or not & $12(27 \%)$ & $10(22 \%)$ & $8(18 \%)$ & $5(11 \%)$ & $6(13 \%)$ & $4(9 \%)$ \\
\hline I probably wouldn't ask & $23(51)$ & $21(47 \%)$ & $20(44 \%)$ & $26(58 \%)$ & $21(47 \%)$ & $27(60 \%)$ \\
\hline I definitely wouldn't ask & $5(11)$ & $4(9 \%)$ & $9(20 \%)$ & $10(22 \%)$ & $11(24.5 \%)$ & $11(24 \%)$ \\
\hline Comparison & \multicolumn{2}{|l|}{$C h i^{2}=2.52, p=0.64$} & \multicolumn{2}{|c|}{$\mathrm{Chi}^{2}=3.01, \mathrm{p}=0.55$} & \multicolumn{2}{|c|}{$\mathrm{Chi}^{2}=6.35, \mathrm{p}=0.17$} \\
\hline \multicolumn{7}{|c|}{ 'How likely is it that you would go ahead with the dental treatment without cover?' } \\
\hline I definitely would & $4(9 \%)$ & $4(9 \%)$ & $6(13 \%)$ & $10(22 \%)$ & $10(22 \%)$ & $13(29 \%)$ \\
\hline I probably would & $15(33 \%)$ & $14(31 \%)$ & $18(40 \%)$ & $20(44 \%)$ & $21(47 \%)$ & $19(42 \%)$ \\
\hline I don't know whether I would or not & $11(25 \%)$ & $14(31 \%)$ & $11(24 \%)$ & $7(16 \%)$ & $4(9 \%)$ & $5(11 \%)$ \\
\hline I probably wouldn't & $10(22 \%)$ & $8(18 \%)$ & $3(7 \%)$ & $5(11 \%)$ & $5(11 \%)$ & $4(9 \%)$ \\
\hline I definitely wouldn't & $5(11 \%)$ & $5(11 \%)$ & $7(16 \%)$ & $3(7 \%)$ & $5(11 \%)$ & $4(9 \%)$ \\
\hline Comparison & \multicolumn{2}{|c|}{$\mathrm{Chi}^{2}=0.62, \mathrm{p}=0.96$} & \multicolumn{2}{|c|}{$C h i^{2}=4.09, p=0.39$} & \multicolumn{2}{|c|}{$C h i^{2}=1.20, p=0.87$} \\
\hline
\end{tabular}

content might justify this result. Patients in the non-video group were most relieved when they received confirmation from a cardiologist who they accepted as a more credible source than a leaflet. This 'credibility' was passed to the patients visually in the video. This demonstrates the importance of consistency of message among health care teams. Luck et $a l^{3}$ assessed the effect of video information on pre-colonoscopy anxiety and knowledge. In this study, an information video increased patients' knowledge and decreased anxiety in patients preparing for colonoscopy. Ruffinengo et al. ${ }^{13}$ highly recommended the application of an informative video, devised for patients undergoing coronarography, as an instrument to be able to lower anxiety levels and increase the level of satisfaction from the received information among patients. In another study leaflets were used to empower patients at consultation and were found to improve patients' satisfaction and perceptions of communication. ${ }^{14}$

Evaluation of a videotape decision-aid to assist patient with prostate cancer in considering treatment options revealed that a videotape decision-aid will benefit clinical practice by conveying knowledge to patients regarding treatment options and outcomes and encouraging them to participate with their physicians in medical decision-making. ${ }^{15}$ A systematic review of RCTs found that communication tools in most formats will increase patients' understanding but are more likely to do so if structured, tailored and/or interactive. ${ }^{16}$ The main limitation of this study was that it was conducted more than a year after the NICE guideline was published and a large group of patients were already aware of the guideline (either from their dentist or their cardiologist). Unfortunately patients' knowledge of the new guideline was not assessed. This could explain the low level of anxiety at baseline. However, they were mostly unaware of the evidence behind the guideline. The other factor expected to limit the accuracy of the study was that this study was conducted in a cardiology department so patients were not actually faced with a real decision on whether to have a dental procedure without antibiotic prophylaxis, which might have affected their level of anxiety and concern.

Moreover, the questionnaire used to assess anxiety and concern regarding dental treatment had not been previously validated. This brings into question the validity of this measure. It is possible that a more sensitive measure may have revealed greater differences.

\section{CONCLUSION}

In conclusion, it appears that communicating evidence of changes in patient care 
through an information video is more likely to reduce patient concerns about the change compared to an information leaflet alone. Clinicians should consider the use of multimedia communication to aid patient care. However, healthcare practitioners have a major influence on patients' acceptance and are able to drive effective implementation of clinical practice guidelines. Indeed, their attitudes towards acceptance or rejection of evidence seem to be reflected in patients' attitudes and can influence patient choice.

\section{PRACTICE IMPLICATIONS}

Communication tools can improve patients' understanding of changes in their care and provide them with necessary information on the pros and cons of different treatment options. It will also encourage them to discuss their concerns with the clinician and coach them on how to ask questions. Accordingly, a health provider will have a chance to offer good-quality counselling and more focused discussions with their patients in a shorter time. Considering the lack of interest in the written material and the probability of low literacy, the present study found benefit from the videotape information developed to help patients on making informed decisions. A further study could assess use of the video one week before the appointment to see if any benefit difference existed.

The authors would like to thank the clinical and nursing staff of the Adult Congenital Heart Disease Service at the St Thomas Hospital for providing the patients for the trial and for the use of facilities. The research described in this paper was presented at the conference of the IADR 2010, Barcelona, where it was winner of the Behavioural Epidemiologic and Health Services Research Outstanding Student Abstract Award. The study was supported by the Ministry of Health and Medical Education, Tehran, Iran as part of the PhD project of the first author (SS) in King's College London.

1. National Institute for Health and Clinical Excellence. Prophylaxis against infective endocarditis: antimicrobial prophylaxis against infective endocarditis in adults and children undergoing interventional procedures. London: NICE, 2008.

2. Epstein, R M, Alper B S, Quill T E. Communicating evidence for participatory decision making. JAMA 2004: 291: 2359-2366.

3. Luck, A, Pearson S, Maddern G, Hewett P. Effect of video information on precolonoscopy anxiety and knowledge: a randomised trial. Lancet 1999; 354: 2032-2035.

4. Hoving C, Visser A, Mullen P D., van den Borne B. A history of patient education by health professionals in Europe and North America: From authority to shared decision making education. Patient Educ Couns 2010; 78: 275-281.

5. Llewellyn-Thomas H A. Patients' health-care decision-making: A framework for descriptive and experimental investigations. Med Decis Making 1995; 15: 101-106.

6. Olver I N, Turrell S J, Olszewski N A, Willson K J.
Impact of an information and consent form on patients having chemotherapy. Med J Aust 1995; 162: 82-83.

7. Stanley B M, Walters D J, Maddern G J. Informed consent: how much information is enough? Aust NZJ Surg 1998; 68: 788-791.

8. Dayer M J, Chambers J B, Prendergast B, Sandoe J A Thornhill M H. NICE guidance on antibiotic prophylaxis to prevent infective endocarditis: a survey of clinicians' attitudes. OIM2013: 106: 237-243.

9. Farook S A, Davis A K, Khawaja N, Sheikh A M. NICE guideline and current practice of antibiotic prophylaxis for high risk cardiac patients (HRCP) among dental trainers and trainees in the United Kingdom (UK). Br Dent J 2012; 213: E6.

10. Soheilipour S, Scambler S, Dickinson C et al. Antibiotic prophylaxis in dentistry: part I. A qualitative study of professionals' views on the NICE guideline. Br Dent J 2011; 211: E1.

11. Ni Riordáin R, McCreary C. NICE guideline on antibiotic prophylaxis against infective endocarditis: attitudes to the guideline and implications for dental practice in Ireland. Br Dent $J$ 2009; 206: E11.

12. Soheilipour S, Scambler S, Dickinson $C$ et al. Antibiotic prophylaxis in dentistry: part II. A qualitative study of patient perspectives and understanding of the NICE guideline. BrDent J2011; 211: E2.

13. Ruffinengo $C$, Versino $E$, Renga G. Effectiveness of an informative video on reducing anxiety levels in patients undergoing elective coronarography: An RCT. Eur J Cardiovasc Nurs 2009; 8: 57-61.

14. Little P, Dorward M, Warner G et al. Randomised controlled trial of effect of leaflets to empower patients in consultation in primary care. Br Med $\mathrm{J}$ 2004; 328: 441-444.

15. Schapira M M, Meade C, Nattinger A B, Enhanced decision-making: the use of a videotape decisionaid for patients with prostate cancer. Patient Educ Couns 1997; 30: 119-127.

16. Trevena L J, Davey H M, Barratt A. Butow P, Caldwell P. A systematic review on communicating with patients about evidence. J Eval Clin Pract 2006: 12: 13-23. 